\title{
Digital Health Platform for Emotional and Self-Management Support of Caregivers of Children Receiving Growth Hormone Treatment
}

\author{
Gabriel SIGNORELLI ${ }^{\mathrm{a}}$, Francisco José NÚÑEZ-BENJUMEA ${ }^{\mathrm{a}}$, Antonio De Arriba \\ MUÑOZ $^{\mathrm{b}}$, Marta Vara CALLAU ${ }^{\mathrm{b}}$, Maitena PIERANTONELLI ${ }^{\mathrm{c}}$, Alba JIMÉNEZ- \\ DÍAZ ${ }^{\mathrm{c}}$ and Luis FERNANDEZ-LUQUE ${ }^{\mathrm{a}, 1}$ \\ a Adhera Health, Inc, Palo Alto, CA, USA \\ ${ }^{\mathrm{b}}$ Pediatric Endocrinology unit, Miguel Servet Children's University Hospital, \\ Zaragoza, Spain \\ ${ }^{c}$ Labpsitec, Universitat de València, Valencia, Spain
}

\begin{abstract}
In recent years there has been growing research on the combination of evidence-based behavioral change techniques with mobile-based recommender systems. In this paper, we have focused on understanding the psychological burdens experienced by caregivers of children undergoing growth hormone treatment $(\mathrm{GHt})$ and the perceived barriers to and drivers of the adoption of a digital health solution. This is a mixed-methods formative research study looking into technical acceptance aspects of using digital health for the emotional support of parents of children undergoing GHt. After one month using the ADHERA CARING platform (Adhera Health, Inc., Palo Alto, CA), individual semi-structured interviews were conducted. ADHERA CARING provides tailored emotional and self-management support to caregivers of children undergoing GHt to improve adherence to treatment through positive education, personalized motivational messages, and emotional support. A preliminary thematic analysis and categorization were carried out, based on the Behavioral Intervention Technology (BIT) model. The majority of participants were female. All caregivers positively valued having the tool, especially at the beginning of treatment. Information provided in the educational module was useful and improved self-efficacy. Motivational messages contributed to commitment and reinforced the educational content, thus promoting continuity of treatment and potentially improving treatment efficacy. Most participants $(n=10,80 \%)$ accessed all educational units and completed all the 27 quiz questions. Regarding the motivational messages, the overall average rating was 4.55 out of 5.00. ADHERA CARING has the potential to help caregivers to understand the treatment journey. Nevertheless, users have identified that some types of educational content are more valuable at specific stages of the treatment journey, which suggests that personalization of educational content is required to adapt to different stages of the patient journey.
\end{abstract}

Keywords. Digital Health, Growth Hormone, Behavioral Intervention, Caregiver

${ }^{1}$ Corresponding Author, Fernandez-Luque, Adhera Health, Inc, Palo Alto, CA, USA; E-mail: luis@adherahealth.com 


\section{Introduction}

Adherence to growth hormone treatment (GHt) among children is variable and remains a problem [1]. Prior research has been looking into digital health tools to support GHt treatment [2], that includes mobile applications [3], and more advanced digital health ecosystems that integrate mobile solutions with connected injection devices [4].

One of the key stakeholders to be supported are the caregivers, normally parents, of children that require long term pharmacological treatment since research shows the negative impact of stress on adherence and overall quality of life [5]. In this paper, we present a study exploring the feasibility of a mobile digital intervention for emotional and self-management support of caregivers of children receiving GHt. The intervention relies on a digital health platform powered by a Health Recommender System [6] that incorporates self-management educational content, motivational messages, and interactive mental well-being content addressing aspects such as stress management, self-esteem [7] and compassion [8].

\section{Methods}

This is a mixed methods formative research study looking into technical acceptance aspects of using digital health for the emotional support to parents of children undergoing GHt. The study was conducted in the Pediatric Endocrinology unit of the Hospital Miguel Servet (Zaragoza, Spain). It received the clearance from the hospital ethical board and the protocol is publicly available at clinicaltrials.gov (NCT04812665).

After a one-month period of using the ADHERA CARING platform (Adhera Health, Inc., Palo Alto, CA), individual semi-structured interviews were conducted. These interviews addressed the psychological burdens experienced by caregivers of children receiving GHt and the barriers and facilitators for the adoption of the mobile solution were discussed in relation to cargivers' individualized needs and expectations related to children's health outcomes.

The interviews were audio recorded and transcribed by one research assistant, and a preliminary thematic analysis and categorization was carried out, based on the Behavioral Intervention Technology Model (BIT) [8], which provides an integrated conceptual and technological framework for eHealth interventions. A preliminary thematic analysis and categorization was carried out, based on the BIT model [8], which provides an integrated conceptual and technological framework for eHealth and mHealth interventions. Demographic and engagement data was also analyzed using descriptive statistics.

\section{1. mHealth-based digital intervention}

ADHERA CARING provides tailored emotional and self-management support to caregivers of children undergoing GHt to improve adherence to treatment through positive education, personalized motivational messages, and emotional support. Once onboarded, users follow an empowerment program with easy-to-digest educational, personalized, and actionable content included for them, and also perform mental wellbeing activities.

Education is provided via different educational pieces of text classified by categories and organized in units. These contents were fully accessible from day one of user 
enrollment. Whenever a unit is tagged as read, its icon turns green to reflect the progress (Figure 1-A). A progress bar is also displayed and advances as the units are tagged as read by the users. Within each category, the last educational unit is a quiz. The quiz is made up of several questions with multiple choices (Figure 1-B). An explanation of the answer is provided right after answering the question. The quiz is only available once all the other units within the category have been marked as read.

Motivational messages are delivered into an inbox in the app (Figure 1-C). Every time the app receives a new message, a push notification is triggered so that the user can access the message directly. Messages can be rated by users with a score from 1 to 5 stars. Messages are delivered by a health recommender system [5]. This algorithm works in two steps. The first step is a knowledge-based algorithm to filter incompatible messages with the user profile. The remaining messages are then passed to a collaborative filtering algorithm, that uses the demographic profile and the user ratings to compute a similarity measurement between the user profile and each message.

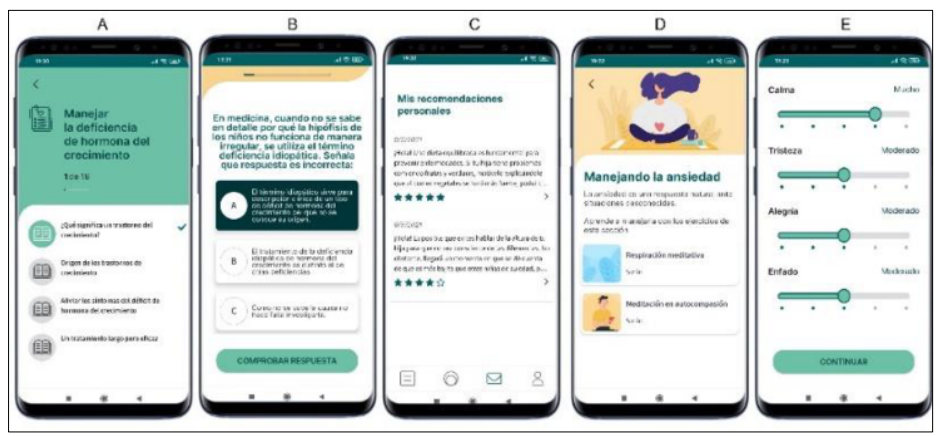

Figure 1. Screenshots of the ADHERA CARING Platform.

The mental well-being module (Figure 1-D) includes two exercises: 'Mindful breathing' and 'Self-compassion meditation'. These are audio-guided sessions that users can complete at their convenience. Before and after each session, the user is asked to complete a well-being assessment (Figure 1-E).

\section{Results}

The majority of participants were female (80\%) with an average age of 45 years. The interviewees positively valued having a tool that provides them with the necessary information and support for emotional well-being to face stressful situations and approach the treatment in a more natural and calm way, avoiding the distress transfer from the caregiver to the child. Notably, many of the caregivers highlighted the importance of having the support of the solution especially at the early stages of treatment.

Regarding the behavior change strategies used to achieve the objectives, the caregivers liked the structure of small topics to facilitate the understanding of each theme. Therefore, the educational content seems to be important to reinforce and remind caregivers of some aspects of patient treatment. The emotional management tips and educational content were also raised as a good initiative. The contents provided by the ADHERA CARING health recommender system (AI based) were perceived useful by the users. Caregivers liked the messages and their frequency. They also found positive 
the strategy to reinforce the educational learnings. In general, the interviewees agreed that ADHERA CARING very intuitive and easy to use, with an attractive design.

Engagement data of the ADHERA CARING platform (Table 1) indicates that $80 \%$ of the total of 10 participants have accessed all 39 available educational units and completed all the 27 available quiz questions. The overall average rating was 4.55 out of 5.00 .

Table 1. Engagement Data

\begin{tabular}{rrrrrr}
\hline Participant & $\begin{array}{c}\text { Education } \\
\text { units } \\
\text { accessed }\end{array}$ & $\begin{array}{c}\text { Education } \\
\text { units } \\
\text { completed }\end{array}$ & $\begin{array}{c}\text { Quiz } \\
\text { Questions } \\
\text { completed }\end{array}$ & $\begin{array}{c}\text { Messages } \\
\text { rated }\end{array}$ & $\begin{array}{c}\text { Average } \\
\text { Rating }\end{array}$ \\
\hline 1 & $39(100 \%)$ & $39(100 \%)$ & $27(100 \%)$ & - & - \\
2 & $39(100 \%)$ & $39(100 \%)$ & $27(100 \%)$ & 17 & 5.00 \\
3 & $39(100 \%)$ & $39(100 \%)$ & $27(100 \%)$ & 41 & 5.00 \\
4 & $39(100 \%)$ & $39(100 \%)$ & $27(100 \%)$ & 46 & 5.00 \\
5 & $39(100 \%)$ & $39(100 \%)$ & $27(100 \%)$ & 32 & 4.03 \\
6 & $39(100 \%)$ & $39(100 \%)$ & $27(100 \%)$ & 6 & 5.00 \\
7 & $39(100 \%)$ & $39(100 \%)$ & $27(100 \%)$ & 36 & 4.11 \\
8 & $39(100 \%)$ & $4(10 \%)$ & $0(0 \%)$ & - & - \\
9 & $39(100 \%)$ & $15(38 \%)$ & $9(33 \%)$ & 6 & 3.33 \\
10 & $39(100 \%)$ & $39(100 \%)$ & $27(100 \%)$ & - & - \\
\hline
\end{tabular}

\section{Conclusions}

The ADHERA CARING platform has potential to help caregivers to understand the treatment journey, specially at the beginning of the GHt, with a friendly interface and easy to digest educational content organized in small units and facilitating the reinforcement of information through personalized messages and quizzes. Nevertheless, users have identified that some types of educational content are more valuable at specific stages of the treatment journey than others, which suggest that deeper personalization adapting to the stage of patient journey is required.

\section{Acknowledgements}

We would like to thank the rest of the team who contributed to this project and the caregivers for their participation. Disclaimer: This investigator-initiated study was financially supported by Merck Healthcare KGaA, Darmstadt Germany (CrossRef Funder ID: 10.13039/100009945). Merck reviewed the manuscript for medical accuracy only before the conference submission. The authors are fully responsible for the content of this manuscript, and the views and opinions described in the publication reflect solely those of the authors.

\section{References}

[1] Haverkamp F, Gasteyger C. A review of biopsychosocial strategies to prevent and overcome earlyrecognized poor adherence in growth hormone therapy of children. Journal of medical economics. 2011 Jan 1;14(4):448-57.

[2] Dimitri P, Fernandez-Luque L, Banerjee I, Bergadá I, Calliari LE, Dahlgren J, de Arriba A, Lapatto R, Reinehr T, Senniappan S, Thomas-Teinturier C. An eHealth Framework for Managing Pediatric Growth 
Disorders and Growth Hormone Therapy. Journal of Medical Internet Research. 2021 May 20;23(5):e27446.

[3] Fernandez-Luque L, Labarta JI, Palmer E, Koledova E. Content analysis of apps for growth monitoring and growth hormone treatment: systematic search in the android app store. JMIR mHealth and uHealth. 2020 Feb 18;8(2):e16208.

[4] Koledova E, Le Masne Q, Spataru A, Bagha M, Dixon D. Digital Health in the Management of Pediatric Growth Hormone Therapy-10 Years of Developments. Studies in Health Technology and Informatics. 2021 May 1;281:926-30.

[5] Armstrong ML, Duncan CL, Stokes JO, Pereira D. Association of caregiver health beliefs and parenting stress with medication adherence in preschoolers with asthma. Journal of Asthma. 2014 May 1;51(4):366-72.

[6] Hors-Fraile S, Malwade S, Luna-Perejon F, Amaya C, Civit A, Schneider F, Bamidis P, Syed-Abdul S, Li YC, De Vries H. Opening the black box: Explaining the process of basing a health recommender system on the i-change behavioral change model. IEEE Access. 2019 Dec 4;7:176525-40.

[7] Germer CK, Neff KD. Self - compassion in clinical practice. Journal of clinical psychology. 2013;69(8):856-867.

[8] Bedrov A, Bulaj G. Improving self-esteem with motivational quotes: opportunities for digital health technologies for people with chronic disorders. Frontiers in psychology 9. 2018:2126.

[9] Mohr DC, Schueller SM, Montague E, Burns MN, Rashidi P. The Behavioral Intervention Technology Model: An Integrated Conceptual and Technological Framework for eHealth and mHealth Interventions. J Med Internet Res 2014;16(6):e146. doi: 10.2196/jmir.3077. 